\title{
Spatiotemporal diversity of macrofungi in the coastal sand dunes of Southwestern India
}

\section{Ghate SD and Sridhar KR*}

Department of Biosciences, Mangalore University, Mangalagangotri, Mangalore 574 199, India

Ghate SD, Sridhar KR 2016 - Spatiotemporal diversity of macrofungi in the coastal sand dunes of Southwestern India. Mycosphere 7(4), 458-472, Doi 10.5943/mycosphere/7/4/6

\begin{abstract}
Spatial and temporal inventory yielded 64 species of macrofungi belonging to 34 genera in five coastal sand dunes (CSDs) of southwest India. Marasmius was the major genus (7 spp.) followed by Agaricus (5 spp.), Lentinus (4 spp.) and Lepiota (4 spp.). Thirty six species were confined to any one of the CSDs, while only Termitomyces striatus distributed in all dunes. Correlation between biotic (species richness, sporocarp richness and diversity) and nine abiotic (air, 2 factors; soil, 7 factors) factors revealed positive correlation of total species $(r=0.396, p=0.05)$ and total exclusive species $(r=0.407, p=0.043)$ against humidity. Sandy soil was preferred substrate of macrofungi (34 spp.) followed by woody substrate (22 spp.). Some preferred twigs, grass and herbivore dung, while others grew on dual substrates (soil/leaf litter; soil/wood; leaf litter/twig). Twenty two species of macrofungi are economically valuable as edible, medicinal and ectomycorrhizal. Amanita sp., Termitomyces schimperi and T. striatus are delicacy for coastal dwellers. Major hindrances for perpetuation of macrofungi in CSDs include sand extraction, wood extraction and clearing vegetation and debris by fire. Preservation of virgin dunes and rehabilitation of degraded dunes will provide ample scope to follow assemblage, divesity and ecological services of macrofungi in coastal habitats.
\end{abstract}

Key words - Abiotic factors - Disturbance - Economic value - Human interference - Mushrooms - Substrate preference

\section{Introduction}

Coastal sand dunes (CSDs) are the intermediary zones serving as interface between terrestrial and marine habitats. They symbolize dynamic ecosystems encompassing baby dunes, dune pastures and dune forests (Høiland 1978). The CSDs are involved in a variety of ecosystem services especially breakers of landward wind velocity, harsh tidal impacts, stabilization of sand and prevention of salt water intrusion (Kidd 2001, Maun 2009, Everard et al. 2010). Although CSDs are highly disturbed habitats, their ecological niches embody and nourish a wide range of biota (Everard et al. 2010). The psammophytes (salt-tolerant vegetation) in CSDs play key role in developing and stabilizing the dunes by their sand-binding potential (Banerjee et al. 2002, Rodrigues et al. 2011). The CSDs are the less comprehended, dynamic and neglected ecosystems with significant knowledge gap on biodiversity (Arun et al. 1999, Rodrigues et al. 2011). However, Sridhar and Bhagya (2007) have drawn attention on economic value of CSD vegetation (as food, fodder and pharmaceuticals) of southwest coast of India. Bhagya and Sridhar (2009) also discussed on the traditional values of coastal sand dune legumes. 
Macrofungi are known to colonize a variety of habitats and serve as saprophytes, decomposers and mycorrhizal in tree species and indirectly facilitating coastal sand dune stability (Pecoraro et al. 2014). Although studies on macrofungi in CSDs are scanty and relatively recent, they are wide spread in different geographic locations (e.g., Brazil, Europe, India, Japan, Seychelles and United States) (Takehashi et al. 2007; Guzmán-Dávalos et al. 2009, Høiland 2012; Hoshino et al. 2009; Akata \& Yaprak 2013, Ghate et al. 2014). Reports of macrofungi in CSDs include gilled fungi (O’Hanlon \& Harrington 2011, Takehashi et al. 2012), ectomycorrhizal fungi (Ashkannejhad \& Horton 2005; Tedersoo et al. 2007, Wolfe et al. 2010; Sulzbacher et al. 2013) and a few new reports (Vila et al. 1999, Takehashi et al. 2007, Hoshino et al. 2009; Taiga et al. 2011, Akata \& Yaprak 2013, Sá et al. 2013). In spite of reports on marine, arbuscular mycorrhizal and endophytic fungi (e.g. Beena et al. 2000, Prasannarai \& Sridhar 2001, Seena \& Sridhar 2004) in the CSDs of southwest India, macrofungal studies are scanty (e.g., Ghate et al. 2014). The previous preliminary survey revealed occurrence of several edible, mycorrhizal and medicinal macrofungi (Ghate et al. 2014). Thus, the current follow up study in CSDs of southwest India envisaged to evaluate macrofungal resource to develop a baseline data: i) by comparison in fairly undisturbed and severely disturbed dunes; ii) to forecast spatiotemporal diversity and distribution pattern; iii) to document substrate preference and economic value.

\section{Materials \& Methods}

\section{Study sites}

The survey in five CSDs was carried out during June-October 2013 at monthly intervals. Five CSDs chosen for study include: Kaup $\left(13^{\circ} 12^{\prime} \mathrm{N}, 74^{\circ} 44^{\prime} \mathrm{E}\right)$, Padubidri $\left(13^{\circ} 12^{\prime} \mathrm{N}, 7^{\circ} 76^{\prime} \mathrm{E}\right)$, Surathkal $\left(12^{\circ} 99^{\prime} \mathrm{N}, 74^{\circ} 79^{\prime} \mathrm{E}\right)$, Thannirbhavi $\left(12^{\circ} 89^{\prime} \mathrm{N}, 74^{\circ} 81^{\prime} \mathrm{E}\right)$ and Someshwara $\left(12^{\circ} 78^{\prime} \mathrm{N}\right.$, $\left.74^{\circ} 85^{\prime} \mathrm{E}\right)$, in west-coast of India. Usually the mid-dunes are covered with sparse vegetation like grasses, sedges, herbs and shrubs. The hind-dunes are predominantly covered by revegetated tree species like Acacia auriculiformis and Casuarina equisetifolia. Besides, some locations consist of natural vegetation like Borassus flabellifer, Erythrina variegata and Pendanus spp. In some locations, plantations of coconut (Cocos nucifera) are established and cultivation of paddy (Oryza sativa) is common beyond hind dunes. In mid- and hind-dunes, deposition of autochthonous and allochthonous organic matter is common (e.g. leaf litter, woody litter, logs, seaweeds, shells and seeds). Mid- and hind-dunes were also covered by above ground dead grasses and sedges. Besides, herbivore grazing resulted in dung accumulation on these CSDs. From each CSD and month, macrofungi exist in $125 \mathrm{~m}^{2}$ plots per sampling were evaluated quantitatively and qualitatively.

\section{Abiotic factors}

Humidity during sampling was determined by Mextech Digital Thermohygrometer (Model \# TM-1; accuracy, $\pm 1 \%$; Mumbai, India) and air temperature (in shade) and soil temperature (10 $\mathrm{cm}$ depth) were measured by mercury thermometer (N.S. Dimple Thermometers, New Delhi, India; Model \# 17876; $\pm 0.2^{\circ} \mathrm{C}$ ). Soil moisture (gravimetric), soil organic carbon (Walkley and Black's rapid titration) and soil nitrogen (macro-Kjeldahl) were estimated following methods outlined by Jackson (1973). Soil pH and electrical conductivity were measured on diluting soil with distilled water (1:2.5 v/v) (Systronics, India, Model \# 304). Additional soil samples were collected to determine the salinity by argentometric method (APHA 1995).

\section{Macrofungi}

Macrofungi grown on the CSDs were assessed for macroscopic characteristics in the field and representative specimen were brought to the laboratory for detailed microscopic examination for identification using monographs (Pegler 1990, Jordan 2004, Phillips 2006, Cannon \& Kirk 2007, Mohanan 2011). The specimens were deposited in the herbarium of the Department of Biosciences, Mangalore University (MUBSSDGKRSCSDMF \# 1-64). 


\section{Data analysis}

The average number of sporocarps of macrofungi in each CSD and month of sampling/quadrat $\left(125 \mathrm{~m}^{2} ; \mathrm{n}=5\right)$ and the overall average sporocarps/quadrat $\left(125 \mathrm{~m}^{2} ; \mathrm{n}=25\right)$ were calculated. Shannon diversity and Pielou's equitability of macrofungi in different CSDs and months were evaluated. Expected number of species, $\left[E_{(s)}\right]$, was calculated based on the number of species versus the number of sporocarps in each CSD and month, the $E_{(s)}$ in a random sample of $n$ sporocarps from a total of $N$ sporocarps was determined. The relationship between richness of species, richness of sporocarps and diversity in CSDs vs. nine abiotic factors was assessed by Pearson correlation ( $p$-values, two-tailed; confidence intervals, $95 \%$ ) (SPSS 16.0: www.spss.com). The Sørensen's similarity coefficient (\%) of macrofungi between CSDs and months was calculated according to Chao et al. (2005).

\section{Results}

Sporocarps of macrofungi which occurred in each CSD irrespective of months and in each month irrespective of CSDs with mean sporocarp data have been presented in Table 1. Species richness, expected number of species, diversity and evenness in CSDs as well as months are presented in Table 2. Altogether 64 macrofungi belonging to 34 genera were recovered in this survey. A total of 36 species was confined to any one of the CSDs as exclusive species. Only Termitomyces striatus was distributed in all CSDs, but none during all months of survey. Of the 34 genera, Marasmius was the major genus (7 spp.) followed by Agaricus (5 spp.), Lentinus (4 spp.) and Lepiota (4 spp.). Photographs of representative species of macrofungi are presented in Figures 1 and 2.

\section{Spatial assemblage}

Among the five CSDs, Someshwara comprises of highest number of species (27 spp.) followed by Thannirbhavi (25 spp.) and Padubidri (24 spp.) (Fig. 3). Average species per quadrat was also highest in Someshwara (8 spp.) followed by Padubidri (5.4 spp.) and Thannirbhavi (5.3 spp.). The sporocarp richness (577) as well as average sporocarp per quadrat (115.4) were highest in Someshwara followed by Thannirbhavi $(343,68.6)$ and Padubidri $(331,66.2)$. Of the 36 exclusive species, Thannirbhavi has the highest $(10 \mathrm{spp}$.) followed by Someshwara/Padubidri ( 8 spp.), Kaup (6 spp.) and Surathkal (4 spp.) (Table 1). Dacryopinax spathularia, Collybia dryophila, Lentinus squarrosulus, Marasmius sp. 2 and Marasmius kisangensis were the top five species distributed in CSDs (2.6-10.4 sporocarps/quadrat). The Shannon diversity was highest in Thannirbhavi followed by Padubidri and Someshwara (Table 2). The rarefaction index of species vs. sporocarps showed the highest expected number of species in the dunes of Someshwara as well as Thannirbhavi (23 spp.) (Fig. 4). Similarity of macrofungi between CSDs ranged between $19.2 \%$ (Thannirbhai vs. Someshwra) and $40 \%$ (Padubidri vs. Thannirbhavi) (Table 3).

\section{Temporal assemblage}

The total as well as average species of macrofungi per quadrat were highest in June (39 and 10 spp.) with a steep decline of total species in July and further recession was gradual, while average species showed a gradual decrease (Fig. 5). Similar to species, total sporocarps and average sporocarps were highest in June (635 and 127 spp.) with a sudden drop in July and decreased gradually thereafter. A total of 39 species was confined to any one of the months with a highest of 19 species in June followed by seven and five species in October and August, respectively (Table 2). The species richness was highest in June (39 spp.) and in rest of the months it ranged between 13 and 19. Shannon diversity was also highest in June. Rarefaction index of species vs. sporocarps showed the highest expected number of species in June than other months (30 vs. 12-18 spp.) (Fig. 4). Similarity of macrofungi between months ranged from $12.5 \%$ (July vs. September) to $45.1 \%$ (August vs. September) (Table 3). 
Table 1 Occurrence of macrofungi in coastal sand dunes of the west coast during monsoon season with overall mean sporocarps per quadrat (Substrate preference: B, bark; D, herbivore dung; G, grass; L, leaf litter; M, termite mound; S, sandy soil; T, twig, W, wood; Importance: *, edible; **, medicinal; ***, ectomycorrhizal).

\begin{tabular}{|c|c|c|c|c|c|c|c|c|c|c|c|c|}
\hline & \multicolumn{10}{|c|}{ Abundance of sporocarps $/ 625 \mathrm{~m}^{2}$ in coastal sand dunes and months } & \multirow{2}{*}{$\begin{array}{l}\text { Sporocarps/ } \\
\text { quadrat } \\
\left(625 \mathrm{~m}^{2}\right) \\
(\text { mean; } \mathbf{n}=25) \\
\end{array}$} & \multirow{2}{*}{$\begin{array}{l}\text { Substrate } \\
\text { preference } \\
\text { and } \\
\text { importance }\end{array}$} \\
\hline & Kaup & $\begin{array}{l}\text { Padu- } \\
\text { bidri }\end{array}$ & $\begin{array}{l}\text { Surath- } \\
\text { kal }\end{array}$ & $\begin{array}{l}\text { Thannir- } \\
\text { bhavi }\end{array}$ & $\begin{array}{l}\text { Somesh- } \\
\text { wara }\end{array}$ & June & July & Aug. & Sept. & Oct. & & \\
\hline \multicolumn{12}{|l|}{ Dacryopinax spathularia } & $\mathrm{W}^{*}$ \\
\hline Collybia dryophila (Bull.) P. & & & & & & & & & & & & $\mathrm{S}$ \\
\hline Kumm. & 1.4 & - & 5.6 & 10.4 & 3.0 & 9.0 & 3.8 & 7.0 & - & 0.6 & 4.1 & \\
\hline Lentinus squarrosulus Mont. & 1.8 & 3.6 & - & 2.0 & 11.2 & - & - & 5.2 & 7.6 & 5.8 & 3.7 & $\mathrm{~W}^{*}$ \\
\hline Marasmius sp. 2 (orange colored) & - & 6.0 & - & 0.6 & 7.0 & - & 6.0 & 0.6 & 7.0 & - & 2.7 & $\mathrm{~L}, \mathrm{~S}$ \\
\hline Marasmius kisangensis Singer & 7.0 & - & - & - & 6.2 & 7.0 & - & 2.2 & 4.0 & - & 2.6 & $\mathrm{~L}$ \\
\hline Crepidotus sp. 1 & - & 2.6 & 2.0 & - & 6.2 & 2.0 & 8.8 & - & - & - & 2.2 & $\mathrm{~T}$ \\
\hline Xylaria hypoxylon (L.) Grev. & - & 1.0 & - & - & - & - & - & - & - & 1.0 & 2.0 & $\mathrm{~W}^{* *}$ \\
\hline Lepiota sp. 1 (orange colored) & - & - & - & 9.6 & - & 8.0 & 1.6 & - & - & - & 1.9 & $\mathrm{~S}$ \\
\hline Marasmius sp. 3 (white) & - & - & 4.0 & - & 5.6 & - & - & 5.6 & 4.0 & - & 1.9 & $\mathrm{~L}, \mathrm{~T}$ \\
\hline Marasmius sp. 1 & - & - & - & 5.4 & 3.4 & 8.8 & - & - & - & - & 1.8 & $\mathrm{~L}, \mathrm{~S}$ \\
\hline Collybia fuscipes (Bull.) Quél. & & 1.0 & 2.2 & 5.4 & - & 6.8 & 1.8 & - & - & - & 1.7 & $\mathrm{~S}, \mathrm{~W}$ \\
\hline Amanita sp. & - & - & - & 4.8 & 3.2 & 7.2 & 0.8 & - & - & - & 1.6 & $\mathrm{~S}^{*}, * *$ \\
\hline Lactarius sp. 1 & 5.0 & 0.6 & 1.2 & 1.2 & - & 5.0 & 1.8 & - & - & 1.2 & 1.6 & $\mathrm{~S}$ \\
\hline Unknown sp. (yellow colored) & 0.6 & - & - & - & 7.6 & 3.4 & 2.0 & 2.8 & - & - & 1.6 & $\mathrm{~S}$ \\
\hline Lycoperdon decipiens Durieu \& & & & & & & & & & & & & $\mathrm{S}^{*}$ \\
\hline Mont. & - & 2.0 & - & 2.2 & 2.4 & 4.4 & - & - & 2.2 & - & 1.3 & \\
\hline Auricularia auricula-judae (Bull.) & & & & & & & & & & & & $\mathrm{W}^{*}$ \\
\hline Quél. & - & 2.2 & - & 4.0 & - & - & - & - & - & 6.2 & 1.2 & \\
\hline Psilocybe sp. & - & 6 & - & - & - & 6.0 & - & - & - & - & 1.2 & $\mathrm{~S}$ \\
\hline Scleroderma citrinum Pers. & - & 1.8 & - & - & 4.0 & 5.0 & 0.8 & - & - & - & 1.2 & $\mathrm{~S} * * *$ \\
\hline Coprinus plicatilis (Curtis) Fr. & - & 2.4 & 2.0 & 1.2 & - & 1.2 & 1.2 & 2.0 & 1.2 & - & 1.1 & $\mathrm{D}$ \\
\hline Crepidotus uber (Berk. \& M.A. & & & & & & & & & & & & $\mathrm{T}$ \\
\hline Curtis) Sacc. & - & 0.8 & - & - & 4.4 & 2.6 & - & 1.8 & 0.8 & - & 1.0 & \\
\hline Lentinus patulus Lév. & - & - & - & - & 5.2 & 5.2 & - & - & - & - & 1.0 & $\mathrm{~W}^{*}$ \\
\hline \multicolumn{13}{|l|}{ Leucocoprinus fragilissimus (Berk. } \\
\hline \& M.A. Curtis) Pat. & - & 5.0 & - & - & - & - & - & - & - & 5.0 & 1.0 & $\mathrm{~S}$ \\
\hline Lenzites betulina (L.) Fr. & - & 1.2 & - & 1.0 & 2.2 & - & - & 1.0 & 3.4 & - & 0.9 & $\mathrm{~W}$ \\
\hline Lenzites sp. 1 & - & 0.4 & 0.8 & - & 3.4 & 1.4 & - & - & 1.6 & 1.6 & 0.9 & $\mathrm{~W}$ \\
\hline Crepidotus reversus (Berk. \& & & & & & & & & & & & & $\mathrm{T}$ \\
\hline Broome) Sacc. & 4.0 & - & - & - & - & 4.0 & - & - & - & - & 0.8 & \\
\hline
\end{tabular}




\begin{tabular}{|c|c|c|c|c|c|c|c|c|c|c|c|c|}
\hline & \multicolumn{10}{|c|}{ Abundance of sporocarps $/ 625 \mathrm{~m}^{2}$ in coastal sand dunes and months } & \multirow{2}{*}{$\begin{array}{l}\text { Sporocarps/ } \\
\text { quadrat } \\
\left(625 \mathbf{~ m}^{2}\right) \\
(\text { mean; } \mathbf{n}=25)\end{array}$} & \multirow{2}{*}{$\begin{array}{l}\text { Substrate } \\
\text { preference } \\
\text { and } \\
\text { importance }\end{array}$} \\
\hline & $\begin{array}{l}\text { Coastal } \\
\text { Kaup }\end{array}$ & $\begin{array}{l}\text { and dun } \\
\text { Padu- } \\
\text { bidri }\end{array}$ & $\begin{array}{l}\text { (mean; n= } \\
\text { Surath- } \\
\text { kal }\end{array}$ & $\begin{array}{l}\text { Thannir- } \\
\text { bhavi }\end{array}$ & $\begin{array}{l}\text { Somesh- } \\
\text { wara }\end{array}$ & $\begin{array}{l}\text { Month } \\
\text { June }\end{array}$ & (mean; 1 & $\begin{array}{l}\text { 1=5) } \\
\text { Aug. }\end{array}$ & Sept. & Oct. & & \\
\hline Lepiota sp. 2 (pink colored) & - & - & 4.0 & - & - & - & - & - & 4.0 & - & 0.8 & $\mathrm{~W}$ \\
\hline Marasmius sp. 5 (found in & & & & & & & & & & & & $\mathrm{S}$ \\
\hline foredune) & - & - & 4.0 & & - & & - & - & - & 4.0 & 0.8 & \\
\hline Agaricus sp. 4 & 1.2 & - & 0.6 & 1.6 & - & - & 3.4 & - & - & - & 0.7 & $\mathrm{~S}$ \\
\hline Ganoderma lucidum (Curtis) P. & & & & & & & & & & & & $\mathrm{W}^{* *}$ \\
\hline Karst. & - & - & 0.6 & 1.2 & 1.8 & 1.6 & 0.8 & 1.2 & - & - & 0.7 & \\
\hline Lepiota sp. 3 (brown coloured) & 0.8 & 2.6 & - & - & - & 1.6 & 1.8 & - & - & - & 0.7 & $\mathrm{~S}$ \\
\hline Termitomyces striatus (Beeli) R. & & & & & & & & & & & & \\
\hline Heim (on termite mound) & 0.2 & 1.0 & 1.0 & 1.2 & 0.2 & 1.6 & 1.8 & - & - & 0.2 & 0.7 & $\mathrm{M}^{*}$ \\
\hline Ganoderma applanatum (Pers.) & & & & & & & & & & & & $\mathrm{W}^{* *}$ \\
\hline Pat. & - & - & - & - & 2.8 & - & - & - & 2.8 & - & 0.6 & \\
\hline Irpex $\mathrm{sp}$. & 1.2 & 1.6 & - & - & - & - & - & - & - & 1.2 & 0.6 & $\mathrm{~W}$ \\
\hline Macrolepiota sp. & - & - & 2.8 & - & - & 2.8 & - & - & - & - & 0.6 & $\mathrm{~S} * * *$ \\
\hline Marasmius spegazzinii (Kuntze) & & & & & & & & & & & & $\mathrm{L}, \mathrm{S}$ \\
\hline Sacc. \& P. Syd. & - & - & - & 3.2 & - & 2.2 & 1.0 & - & - & - & 0.6 & \\
\hline Lentinus polychrous Lév. & - & - & - & 3.2 & - & - & - & 3.2 & - & - & 0.6 & $\mathrm{~W}$ \\
\hline Thelephora palmata (Scop.) Fr. & - & - & - & 3.0 & - & - & - & 3.0 & - & - & 0.6 & $\mathrm{~S} * * *$ \\
\hline Daedalia sp.1 & 1.4 & - & 1.2 & - & - & 1.4 & - & 1.2 & - & - & 0.5 & $\mathrm{~W}$ \\
\hline Lepiota clypeolaria (Bull.) P. & & & & & & & & & & & & $\mathrm{S}$ \\
\hline Kumm. & - & - & - & 2.4 & - & 2.4 & - & - & - & - & 0.5 & \\
\hline Agaricus sp3 & - & - & - & 2.0 & - & - & - & 2.0 & - & - & 0.4 & $\mathrm{~S}$ \\
\hline Hygrocybe astatogala $\mathrm{R}$. Heim ex & & & & & & & & & & & & $\mathrm{S}$ \\
\hline $\begin{array}{l}\text { Heinem. } \\
\text { Hed }\end{array}$ & 2.0 & - & - & - & - & - & - & 2.0 & - & - & 0.4 & \\
\hline Macrolepiota dolichaula (Berk. \& & & & & & & & & & & & & \\
\hline Broome) Sacc. & - & - & - & 1.0 & 0.8 & - & - & - & - & 1.8 & 0.4 & $\mathrm{~S} *, * * *$ \\
\hline Termitomyces schimperi (Pat.) R. & & & & & & & & & & & & \\
\hline Heim. (on termite mound) & 2.0 & - & - & - & - & 2.0 & - & - & - & - & 0.4 & $\mathrm{M}^{*}$ \\
\hline Conocybe apala (Fr.) Arnolds (in & & & & & & & & & & & & G \\
\hline grass) & 1.6 & - & - & - & - & - & 1.6 & - & - & - & 0.3 & \\
\hline Conocybe sp. 1 & - & - & - & 1.6 & - & - & 1.6 & - & - & - & 0.3 & $\mathrm{~S}$ \\
\hline Lycoperdon utriforme Bull. & - & - & - & - & 1.4 & 1.4 & - & - & - & - & 0.3 & $\mathrm{~S}^{*}, * *$ \\
\hline Pisolithus sp. & - & - & - & - & 1.6 & 1.6 & - & - & - & - & 0.3 & $\mathrm{~S} * * *$ \\
\hline Polyporus arcularius Rostk. & - & - & 0.8 & - & 0.8 & - & - & - & 1.6 & - & 0.3 & $\mathrm{~W}$ \\
\hline Agaricus sp. 2 & - & 1.0 & - & - & - & - & - & - & 1.0 & - & 0.2 & $\mathrm{~S}$ \\
\hline Cystoagaricus trisulphuratus & & & & & & & & & & & & $\mathrm{S}$ \\
\hline (Berk.) Singer & - & - & - & - & 1.0 & 0.2 & - & 0.8 & - & - & 0.2 & \\
\hline Hexagonia tenuis Speg. & - & 1.2 & - & - & - & - & - & - & - & 1.2 & 0.2 & W \\
\hline
\end{tabular}




\begin{tabular}{|c|c|c|c|c|c|c|c|c|c|c|c|c|}
\hline & & \multirow{3}{*}{$\begin{array}{l}\text { Sporocarps/ } \\
\text { quadrat } \\
\left(625 \mathrm{~m}^{2}\right) \\
(\text { mean; } \mathbf{n}=25)\end{array}$} & \multirow{3}{*}{$\begin{array}{l}\text { Substrate } \\
\text { preference } \\
\text { and } \\
\text { importance }\end{array}$} \\
\hline & \multicolumn{5}{|c|}{$\begin{array}{l}\text { Abundance of sporocarps } / 625 \mathrm{~m}^{-} \text {in coastal sand dunes } \\
\text { Coastal sand dune (mean; } n=5 \text { ) }\end{array}$} & \multicolumn{5}{|c|}{ Month (mean; $\mathbf{n = 5}$ ) } & & \\
\hline & Kaup & $\begin{array}{l}\text { Padu- } \\
\text { bidri }\end{array}$ & $\begin{array}{l}\text { Surath- } \\
\text { kal }\end{array}$ & $\begin{array}{l}\text { Thannir- } \\
\text { bhavi }\end{array}$ & $\begin{array}{l}\text { Somesh- } \\
\text { wara }\end{array}$ & June & July & Aug. & Sept. & Oct. & & \\
\hline Hydnellum sp. 1 & - & - & - & 1.2 & - & - & - & 1.2 & - & - & 0.2 & $\mathrm{~W}$ \\
\hline Macrolepiota rhacodes (Vittad.) & & & & & & & & & & & & $\mathrm{S} *, * * *$ \\
\hline Singer & 0.8 & - & - & - & - & 0.8 & - & - & - & - & 0.2 & \\
\hline Pisolithus albus Alb. \& Schwein. & - & 1.0 & - & - & - & 1.0 & - & - & - & - & 0.2 & $\mathrm{~S} * * *$ \\
\hline Agaricus sp. 1 & - & - & - & 0.6 & - & 0.6 & - & - & - & - & 0.1 & $\mathrm{~S}$ \\
\hline Cystolepiota sp. & - & 0.6 & - & - & - & 0.6 & - & - & - & - & 0.1 & $\mathrm{~S}$ \\
\hline Daedalea flavida Lév. & - & - & - & - & 0.6 & - & - & - & - & 0.6 & 0.1 & $\mathrm{~W}$ \\
\hline Ganoderma sp. & - & - & 0.4 & - & - & 0.4 & - & - & - & - & 0.1 & $\mathrm{~W}^{* *}$ \\
\hline Lentinus sp. 2 & 0.4 & - & - & - & - & 0.4 & - & - & - & - & 0.1 & $\mathrm{~W}$ \\
\hline Marasmius sp. 4 & - & - & - & - & 0.4 & - & 0.4 & - & - & - & 0.1 & $\mathrm{~L}, \mathrm{~S}$ \\
\hline Microporus xanthopus (Fr.) Kuntze & - & 0.6 & - & - & - & 0.6 & - & - & - & - & 0.1 & $\mathrm{~W}^{* *}$ \\
\hline Pleurotus flabellatus (Berk. \& & & & & & & & & & & & & $\mathrm{W}^{*}$ \\
\hline Broome) Sacc. & - & - & - & 0.4 & - & 0.4 & - & - & - & - & 0.1 & \\
\hline Agaricus caribaeus Pegler & - & - & - & - & 0.2 & 0.2 & - & - & - & - & $<0.1$ & $\mathrm{~S}$ \\
\hline Lycoperdon sp. & 0.2 & - & - & - & - & 0.2 & - & - & - & - & $<0.1$ & $\mathrm{~S}$ \\
\hline
\end{tabular}

Table 2 Species richness, diversity and evenness of macrofungi in different coastal sand dunes and months in the Western coast (*Expected number of species out of 125 random number of sporocarps).

\begin{tabular}{|c|c|c|c|c|c|}
\hline \multirow{2}{*}{$\begin{array}{l}\text { Sand dune/ } \\
\text { Season }\end{array}$} & \multicolumn{3}{|c|}{ Species richness } & \multirow{2}{*}{$\begin{array}{l}\text { Shannon } \\
\text { diversity }\end{array}$} & \multirow{2}{*}{$\begin{array}{l}\text { Pielou's } \\
\text { evenness }\end{array}$} \\
\hline & Total & $E_{(s 125)} *$ & Exclusive & & \\
\hline \multicolumn{6}{|l|}{ Sand dune } \\
\hline Kaup & 18 & 17 & 6 & 3.649 & 0.875 \\
\hline Padubidri & 24 & 22 & 8 & 4.032 & 0.879 \\
\hline Surathkal & 17 & 17 & 4 & 3.603 & 0.881 \\
\hline Thannirbhavi & 25 & 23 & 10 & 4.164 & 0.897 \\
\hline Someshwara & 27 & 23 & 8 & 3.946 & 0.830 \\
\hline \multicolumn{6}{|l|}{ Month } \\
\hline June & 39 & 30 & 19 & 4.700 & 0.889 \\
\hline July & 19 & 18 & 4 & 3.371 & 0.794 \\
\hline August & 18 & 17 & 5 & 3.471 & 0.832 \\
\hline September & 13 & 13 & 4 & 3.404 & 0.920 \\
\hline October & 13 & 12 & 7 & 3.165 & 0.855 \\
\hline
\end{tabular}



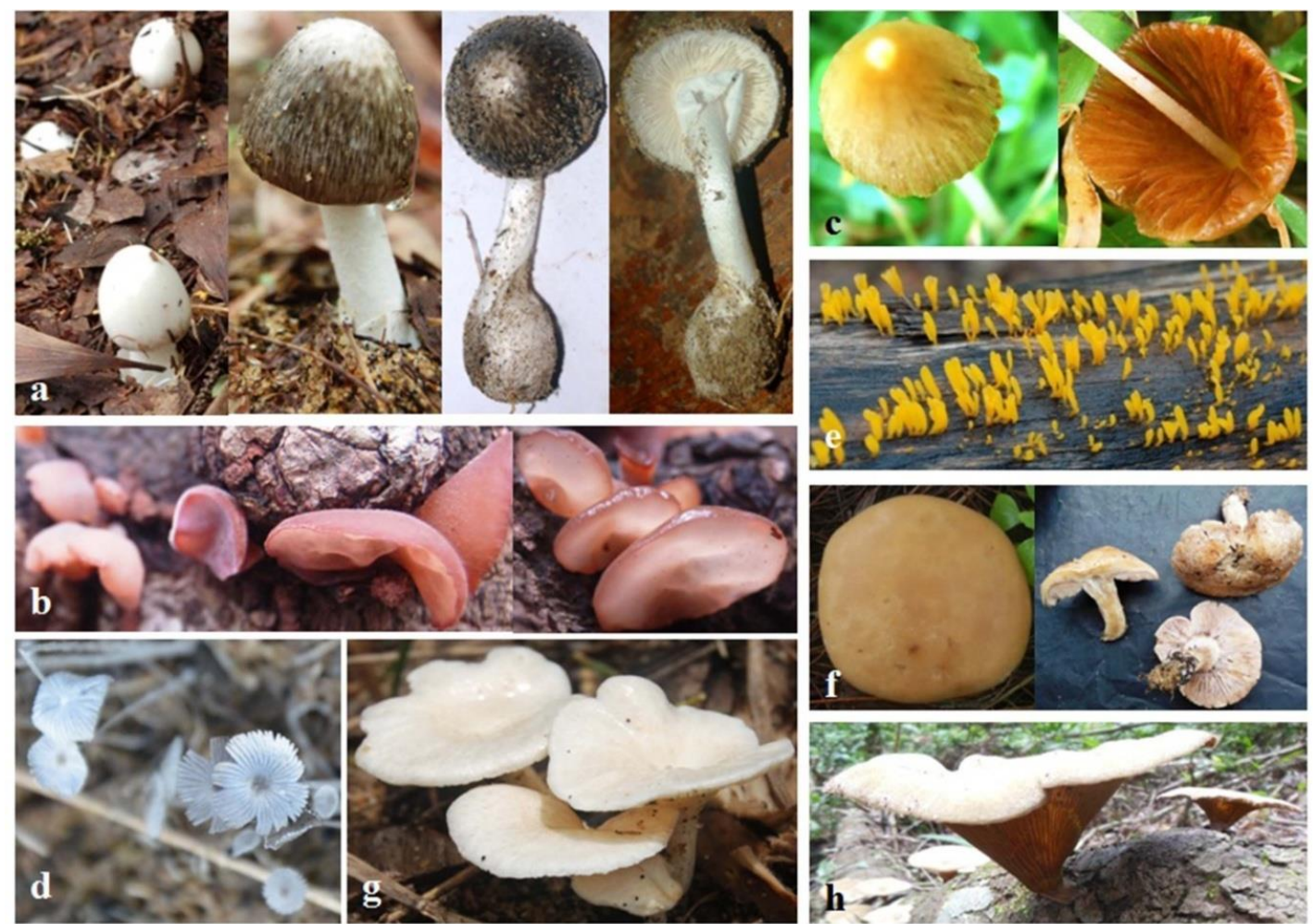

Fig. 1 - Sporocarps of selected macrofungi: a, Amanita sp.; b, Auricularia auricula-judae; c, Conocybe apala; d, Coprinus plicatilis; e, Dacryopinax spathularia; f, Lactarius sp.; g, Lentinus patulus; h, Lentinus polychrous.
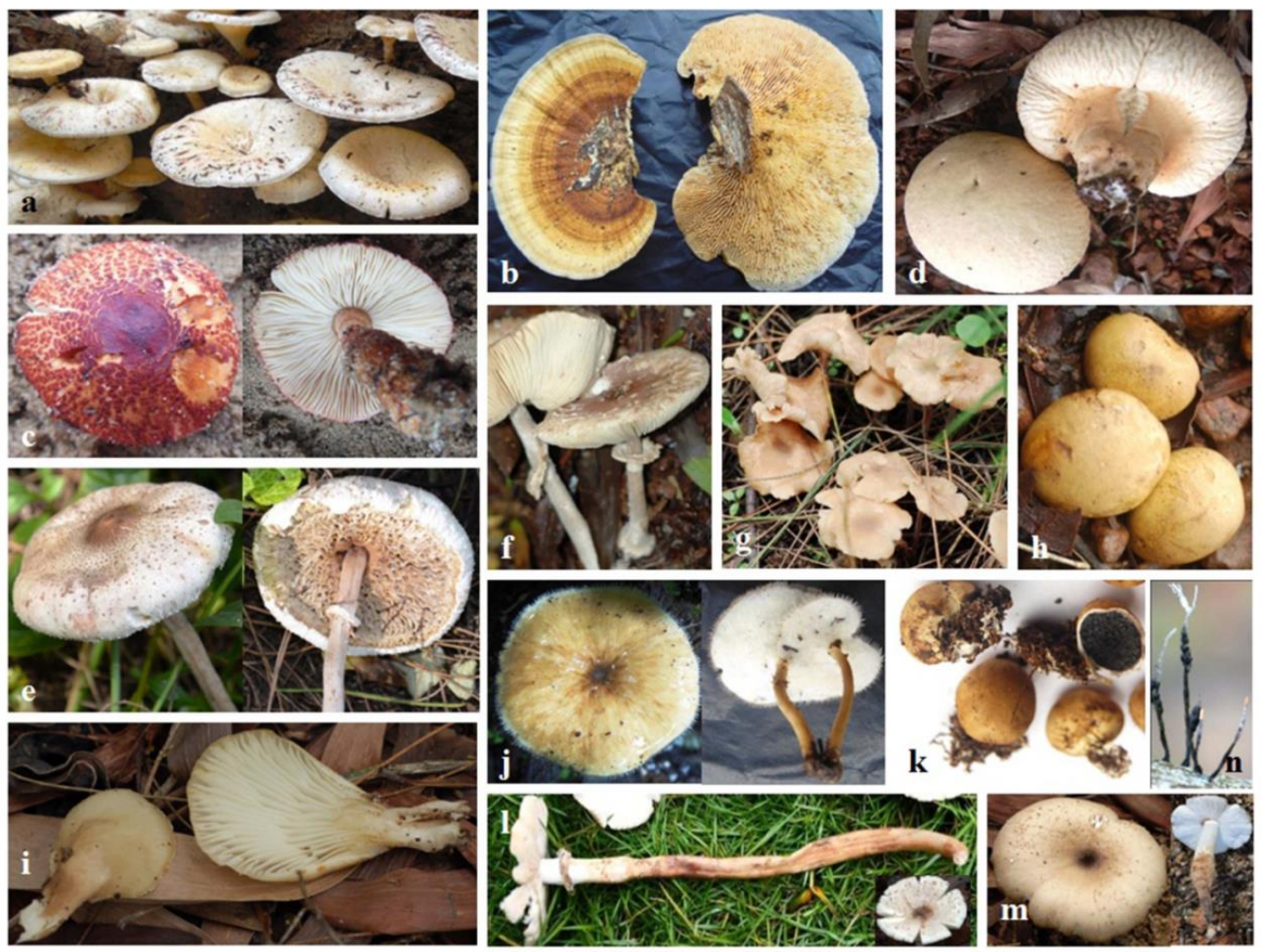

Fig. 2 - Sporocarps of selected macrofungi: a, Lentinus squarrosulus; b, Lenzites betulina; c, Lepiota sp.1; d, Lycoperdon utriforme; e, Macrolepiota dolichaula; f, Macrolepiota rhacodes; g, Marasmius confertus; h, Pisolithus albus; i, Pleurotus flabellatus.; j, Polyporus arcularius; k, Scleroderma citrinum; 1 , Termitomyces schimperi; $\mathrm{m}$, Termitomyces striatus; $\mathrm{n}$, Xylaria hypoxylon. 
Table 3 Sørensen's similarity (\%) of macrofungal species in coastal sand dunes and months.

\begin{tabular}{ccccc}
\hline Sand dunes & & & & \\
\multirow{4}{*}{ Kaup } & Padubidri & Surathkal & Thannirbhavi & Someshwara \\
& 28.6 & 34.3 & 23.3 & 26.7 \\
& Padubidri & 34.1 & 40.0 & 39.2 \\
& & Surathkal & 33.4 & 36.4 \\
Month & & & Thannirbhavi & 19.2 \\
\hline \multirow{2}{*}{ June } & July & August & September & October \\
& \multirow{2}{*}{44.8} & 31.6 & 19.2 & 15.4 \\
& July & 32.4 & 12.5 & 18.6 \\
& & August & 45.1 & 12.9 \\
& & & September & 15.4 \\
\hline
\end{tabular}

\begin{abstract}
Abiotic factors
During the survey, air and soil temperatures were the lowest in Surathkal dunes, whereas they were highest in dunes of Thannirbhavi (Table 4). Humidity was more or less in uniform range $(86.8-87.8 \%)$. Due to sandy texture, moisture was as low as $9.2-15.4 \%$, while $\mathrm{pH}$ was acidic in three dunes (6.6-6.9) and alkaline in two dunes (7.3). Soil conductivity was low ranging between 0.2 and $0.7 \mathrm{mS} / \mathrm{cm}$, while salinity ranged between 0.1 and $0.5 \mathrm{psu}$. The organic carbon was highest in Someshwara (4.9\%) and lowest in Surathkal (1.9\%), while soil nitrogen ranged between 1.1 and $1.4 \%$.

The air temperature was highest during October and lowest during September. The humidity was lowest during October $(80.6 \%)$ and highest in June $(91.8 \%)$. The soil temperature was lowest in July and highest in June (26.3 vs. $27.2 \%$ ), while it was opposite for soil moisture (16.6 vs. $9.4 \%$ ). Soil pH was acidic in September (6.3) and alkaline in June (7.9). Soil conductivity ranged between 0.1 (August) and $0.7 \mathrm{mS} / \mathrm{cm}$ (June-July), while soil salinity 0.04 (September) and $0.6 \mathrm{psu}$ (June). The soil organic carbon was highest during September (5.7 \%) and it was lowest during August (1.7\%), while the soil nitrogen was highest during October (2.5\%) and lowest in August $(0.9 \%)$.

Pearson correlation between three biotic (species richness, sporocarp richness and diversity) and nine abiotic (air and soil) factors revealed varied results. Only total species $(r=0.396, p=$ $0.05)$ and total exclusive species $(r=0.407, p=0.043)$ showed positive correlation against humidity.
\end{abstract}

\title{
Substrate preference and economic value
}

Based on substrate preference, sandy soil (34 spp.) was most preferred followed by woody litter (22 spp.) and leaf litter (6 spp.) (Table 1; Fig. 6a). Besides these substrates, some macrofungi preferred twigs, grass and dung. Some grew on dual substrates like soil and leaf litter, soil and wood and leaf litter and twig. Two species preferred to grow on termite mounds. Up to 12 species in CSDs were edible, seven species each were medicinal and ectomycorrhizal. Two species each were edible + medicinal and edible + ectomycorrhizal (Table 1; Fig. 6b).

\section{Discussion}

\section{Spatial and temporal diversity}

Compared to other parts of the world, there are a few reports on the macrofungi in coastal regions of India (e.g. mangroves: Dutta et al. 2013, CSDs: Ghate et al. 2014). Assemblage of macrofungi in Sundarban mangroves of east coast of India (62 spp. in 46 genera) matches with the present study (Dutta et al. 2013). Dacryopinax spathularia was dominant in the mangroves of Southwest India as well as Sundarbans (Dutta et al. 2013, Ghate et al. 2014). The mangroves close to the CSDs surveyed consist of low macrofungal assemblage (46 spp. in 27 genera) (Ghate \& Sridhar, 2015). Studies on macrofungi in plantations and botanical garden of lateritic terrain of the 

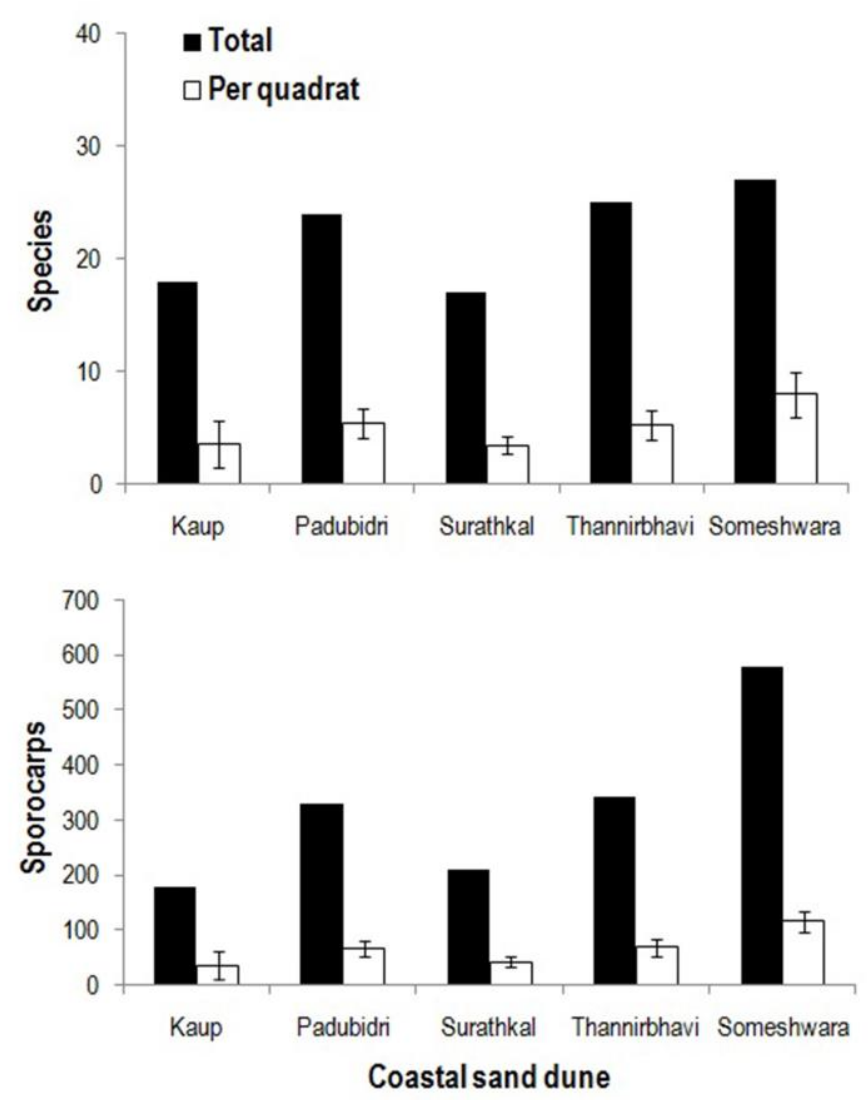

Fig. 3 - Total species, species per quadrat $(n=5 \pm S E)$, total sporocarps and sporocarps per quadrat $(n=5 \pm$ $\mathrm{SE})$ in five coastal sand dunes.
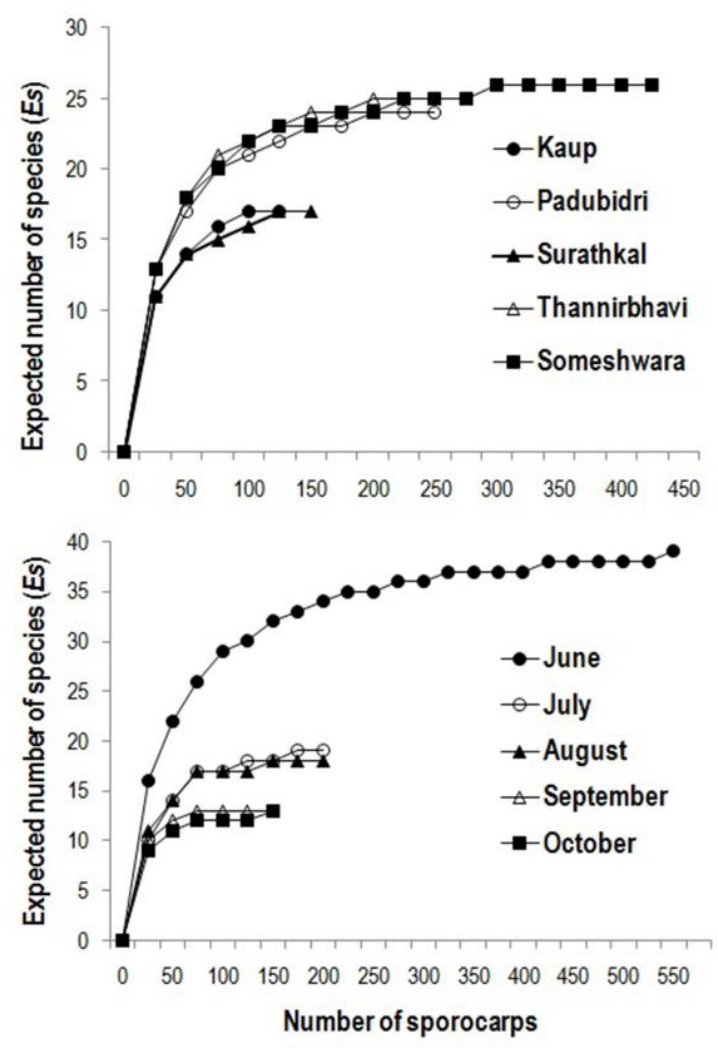

Fig. 4 - Rarefaction curves of macrofungal expected number of species $\left[E_{(\mathrm{s})}\right]$ against sporocarp abundance in different coastal sand dunes and months. 

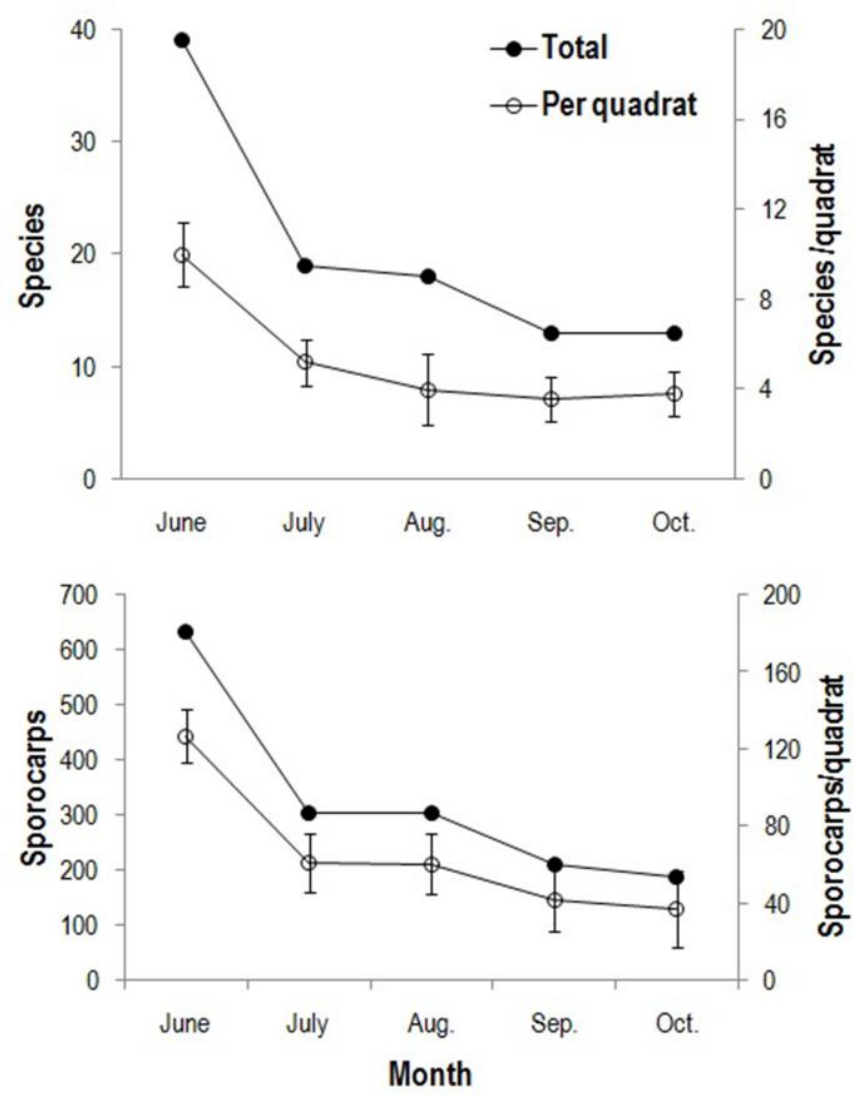

Fig. 5 - Total species, species per quadrat $(n=5 \pm S E)$, total sporocarps and sporocarps per quadrat $(n=5 \pm S E)$ in different months.
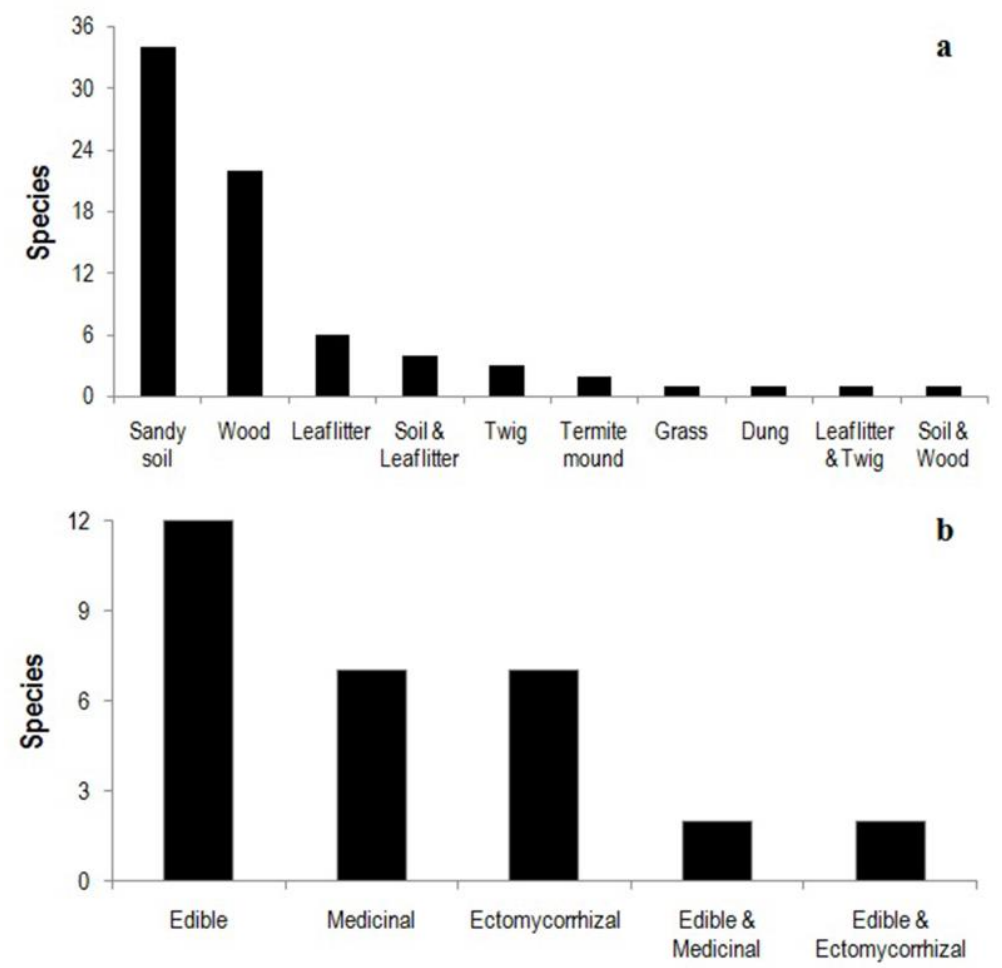

Fig. 6 - Occurrence of macrofungi in different substrates in the coastal sand dunes (a) and their economic value (b). 
Table 4 Fluctuation in air and soil edaphic factors in coastal sand dunes and during June-October $(n=5 \pm S D)$.

\begin{tabular}{|c|c|c|c|c|c|c|c|c|c|c|}
\hline & \multicolumn{5}{|c|}{ Coastal sand dune } & \multicolumn{5}{|l|}{ Month } \\
\hline & Kaup & Padubidri & Suratkal & $\begin{array}{l}\text { Thannir- } \\
\text { bhavi }\end{array}$ & $\begin{array}{l}\text { Somesh- } \\
\text { wara }\end{array}$ & June & July & August & September & October \\
\hline Humidity (\%) & $87.8 \pm 1.8$ & $87.6 \pm 1.0$ & $87.0 \pm 3.4$ & $86.8 \pm 2.0$ & $87.2 \pm 2.6$ & $91.8 \pm 1.7$ & $91.6 \pm 0.8$ & $84.6 \pm 0.8$ & $87.8 \pm 1.4$ & $80.6 \pm 1.5$ \\
\hline $\begin{array}{l}\text { Air temperature } \\
\left({ }^{\circ} \mathrm{C}\right)\end{array}$ & $26.3 \pm 0.6$ & $25.3 \pm 0.4$ & $25.1 \pm 0.9$ & $26.3 \pm 1.1$ & $25.9 \pm 0.6$ & $25.7 \pm 1.1$ & $24.9 \pm 0.4$ & $26.7 \pm 0.4$ & $24.3 \pm 0.5$ & $27.2 \pm 0.6$ \\
\hline $\begin{array}{l}\text { Soil temperature } \\
\left({ }^{\circ} \mathrm{C}\right)\end{array}$ & $27.2 \pm 0.4$ & $26.8 \pm 0.4$ & $26.2 \pm 0.2$ & $27.3 \pm 0.7$ & $26.8 \pm 0.2$ & $27.2 \pm 0.8$ & $26.3 \pm 0.3$ & $27.1 \pm 0.4$ & $26.5 \pm 0.4$ & $27.1 \pm 0.4$ \\
\hline $\begin{array}{l}\text { Soil moisture } \\
(\%)\end{array}$ & $13.1 \pm 1.6$ & $9.2 \pm 2.3$ & $15.4 \pm 1.4$ & $14.17 \pm 1.8$ & $14.4 \pm 2.7$ & $9.4 \pm 1.8$ & $16.6 \pm 1.2$ & $16.2 \pm 1 . .7$ & $14.6 \pm 2.2$ & $9.5 \pm 2$ \\
\hline Soil pH & $7.3 \pm 0.1$ & $6.8 \pm 0.3$ & $7.3 \pm 0.3$ & $6.9 \pm 0.4$ & $6.6 \pm 0.2$ & $7.9 \pm 0.2$ & $7.3 \pm 0.2$ & $6.6 \pm 0.1$ & $6.3 \pm 0.1$ & $6.7 \pm 0.2$ \\
\hline $\begin{array}{l}\text { Soil conductivity } \\
(\mathrm{mS} / \mathrm{cm})\end{array}$ & $0.2 \pm 0.05$ & $0.5 \pm 0.3$ & $0.7 \pm 0.3$ & $0.5 \pm 0.2$ & $0.4 \pm 0.09$ & $0.7 \pm 0.1$ & $0.7 \pm 0.1$ & $0.1 \pm 0.001$ & $0.2 \pm 0.01$ & $0.6 \pm 0.1$ \\
\hline $\begin{array}{l}\text { Soil salinity } \\
\text { (psu) }\end{array}$ & $0.1 \pm 0.04$ & $0.5 \pm 0.2$ & $0.4 \pm 0.01$ & $0.3 \pm 0.1$ & $0.2 \pm 0.07$ & $0.6 \pm 0.01$ & $0.3 \pm 0.01$ & $0.1 \pm 0.001$ & $0.04 \pm 0.01$ & $0.5 \pm 0.05$ \\
\hline $\begin{array}{l}\text { Soil organic } \\
\text { carbon }(\%)\end{array}$ & $2.0 \pm 0.4$ & $3.7 \pm 1.2$ & $1.9 \pm 0.11$ & $3 \pm 0.9$ & $4.9 \pm 0.9$ & $1.9 \pm 0.2$ & $1.8 \pm 0.2$ & $1.7 \pm 0.2$ & $5.7 \pm 0.9$ & $4.4 \pm 0.7$ \\
\hline $\begin{array}{l}\text { Soil nitrogen } \\
(\%)\end{array}$ & $1.1 \pm 0.2$ & $1.2 \pm 0.2$ & $1.4 \pm 0.3$ & $1.4 \pm 0.5$ & $1.3 \pm 0.2$ & $1.0 \pm 0.1$ & $1.0 \pm 0.2$ & $0.9 \pm 0.1$ & $1.0 \pm 0.2$ & $2.5 \pm 0.3$ \\
\hline
\end{tabular}


southwest India (79 spp. in 53 genera) and Western Ghats forests (157 spp. in 87 genera) (Karun \& Sridhar 2014, 2016) showed higher assemblage than the present study. Assemblages in the sand dunes of Welsh (total, 502 spp.; agarics, 441 spp.) (Pegler et al. 1993), Norway (75 spp.) (Høiland 2012) and Italy (160 spp.) (Lantieri et al. 2009) were also higher than present study.

Month of June is highly productive in macrofungal sporocarps as well as diversity corroborating with surveys in mangroves and lateritic region of the southwest coast (Karun \& Sridhar 2014, Ghate \& Sridhar 2015, Pavithra et al. 2016). Temporal studies resulted in two peaks of macrofungal sporocarp diversity (June and September) in the Western Ghats (Karun \& Sridhar 2016) may be due to fluctuations in abiotic factors. In our study, dunes with less disturbance (Padubidri, Thannirbhavi and Someshwara) showed higher assemblages than those with severe disturbance (Kaup and Surathkal) as seen in rarefaction curves. Unfortunately, there are no reports from the virgin dunes of southwest India for comparison.

\section{Biotic and abiotic factors}

The CSDs of southwest India are facing several human interferences (e.g. fishing, recreation and sand mining). Typical flora in dunes include mat-forming creepers, herbs, grasses, sedges, climbers, scrubs and trees (Sridhar, 2009). Complex vegetation with their root system prevents sand erosion and supports mycorrhizal fungi. In addition, leaf litter and dead roots serve as major source of organic matter. Tree species in the hind-dunes and beyond are useful as they provide canopy cover, which controls abiotic and edaphic conditions of dunes. The total and exclusive species of macrofungi showed positive correlation with humidity. The humidity in June was highest $(91.8 \%)$ and might be ideal for growth of macrofungi as there were many exclusive species (19 spp.). Detailed week-wise inventory during June-July may result in tracing the impact of abiotic factors more precisely.

\section{Substrate preference and economic value}

Logs, leaf/woody litter, twigs, seeds, seaweeds and shells are common substrates on the mid- and hind-dunes. Autochthonous and allochthonous organic debris composed of mainly leaf/woody litter, twigs and grass shreds. In addition, grazing by stray cattle results in accumulation of herbivore dung on the dunes. Organic matter with herbivore dung results in appareance of macrofungi. Our study showed highest macrofungi on sandy soil (34 spp.) followed by woody litter (22 spp.). Interestingly, six macrofungi preferred more than one substrate. However, in mangrove habitats, assemblage of macrofugi was higher on the woody litter (26 spp.) than soil (22 spp.) probably due to inundation (Ghate \& Sridhar 2015).

Nearly $47 \%$ of macrofungi encountered in our study are of some economic value. Amanita sp., Termitomyces schimperi and T. striatus are delicacies of coastal dwellers. Interestingly, $T$. striatus was distributed in all CSDs surveyed. Up to seven macrofungi in dunes were of medicinal value and likely to produce secondary metabolites due to stressed conditions (e.g. salintiy, alkaline $\mathrm{pH}$ and sand abbression). Seven species were ectomycorrhizal with Acacia auriculiformis and Casuarina equisitifolia. Ectomycorrhizal fungi were also reported from CSDs of other parts of the world (e.g. Amanita, Boletus, Coltricia, Hygrocybe, Lactarius, Lactifluus, Russula, Scleroderma, Tylopilus and Typhula) (Cibula \& Weber 1996, Miller et al. 2000, Ashkannejhad \& Horton 2005, Tedersoo et al. 2007, Hoshino et al. 2009, Wolfe et al. 2010, Sulzbacher et al. 2013). Future studies on ectomycorrhizal fungi with native tree species in dunes helpful for aforestation. Human interference is one of major threats for the CSDs worldwide (e.g. urbanization, road construction, building seawall, recreation and deforestation). Main threats for CSDs in the southwest of India include sand extraction, wood extraction and setting fire to remove vegetation/organic debris, which severely hinder macrofungal perpetuation.

This the first inventory on the macrofungi in CSDs of India and thus will form a baseline data. Conservation of virgin dunes and rehabilitation of degraded dunes will provide opportunities to understand assemblage, divesity, benefits and ecological services of macrofungi, in future. 


\section{Acknowledgements}

Authors are grateful to Mangalore University for permission to carry out this study in the Department of Biosciences. SDG acknowledges the award of an INSPIRE Fellowship by the Department of Science and Technology, New Delhi (DST/INSPIRE Fellowship/2013/132: Award \# IF130237). KRS acknowledges the award of UGC-BSR Faculty Fellowship by the University Grants Commission, New Delhi. The authors thank Dr. N. Karun Chinnappa for assistance in identification of macrofungi and the referee for improving the clarity of the manuscript. The authors declare that there are no competing interests to publish this paper in Mycosphere.

\section{References}

Akata I, Yaprak AE. 2013 - A new Peziza record for Turkish mycobiota. Biological Diversity and Conservation 6, 32-34.

APHA. 1995 - Standard Methods in Examination of Water and Waste Water (19th Edition). American Public Health Association, Washington DC.

Arun AB, Beena KR, Raviraja NS, Sridhar KR. 1999 - Coastal sand dunes - A neglected ecosystem. Current Science 77, 19-21.

Ashkannejhad S, Horton TR. 2005 - Ectomycorrhizal ecology under primary succession on coastal sand dunes: Interactions involving Pinus contorta, suilloid fungi and deer. New Phytologist 169, 345-354.

Banerjee LK, Rao TA, Sastry ARK, Ghosh D. 2002 - Diversity of Coastal Plant Communities in India. Ministry of Environment and Forests, Botanical Survey of India Kolkata, India.

Beena KR, Raviraja NS, Arun AB, Sridhar KR. 2000 - Diversity of arbuscular mycorrhizal fungi on the coastal sand dunes of the west coast of India. Current Science 79,1459-1466.

Bhagya B, Sridhar KR, 2009 - Ethnobiology of coastal sand dune legumes of southwest India. Indian Journal of Traditional Knowledge 9, 611-620.

Cannon PF, Kirk PM. 2007 - Fungal Families of the World. CAB International, UK.

Chao A, Chazdon RL, Colwell RK, Shen T-J. 2005 - A new statistical approach for assessing similarity of species composition with incidence and abundance data. Ecology Letters $8,148-159$.

Cibula WG, Weber NS. 1996 - Hygrocybe andersonii a new psammophilus Hygrocybe from Horn Island, a Mississippi Barrier Island. Mycologia 88, 514-516.

Dutta AK, Pradhan P, Basu SK, Acharya K. 2013 - Macrofungal diversity and ecology of the mangrove ecosystem in the Indian part of Sundarbans. Biodiversity 14,196-206.

Everard M, Jones L, Watts B. 2010 - Have we neglected the societal importance of sand dunes? An ecosystem services perspective. Aquatic Conservation: Marine and Freshwater Ecosystems 20, 476-487.

Ghate SD, Sridhar KR, Karun NC. 2014 - Macrofungi on the coastal sand dunes of South-western India. Mycosphere 5, 144-151.

Ghate SD, Sridhar KR. 2015 - Contribution to the knowledge on macrofungi in mangroves of the Southwest India. Plant Biosystems; doi: 10.1080/11263504.2014.994578

Guzmán-Dávalos L, Ortega A, Contu M, Vizzini A, Rodríguez A, Villalobos-Arámbula AR, Santerre A. 2009 - Gymnopilus maritimus (Basidiomycota, Agaricales), a new species from coastal psammophilous plant communities of northern Sardinia, Italy, and notes on G. arenophilus. Mycological Progress 8, 195-205.

Høiland K, 1978 - Sand-dune vegetation of Lista, SW Norway. Norwegian Journal of Botany 25, 23-45.

Høiland K. 2012 - An investigation of basidiospore characteristics in sand dune mushrooms from Lista, South-Western Norway. Agarica 32, 49-58.

Hoshino T, Takehashi S, Fujiwara M. 2009 - Typha maritima, a new species of Typhula collected from coastal dunes in Hokkaido, Northern Japan. Mycoscience 50, 430-437.

Jackson ML. 1973 - Soil Chemical Analysis. Prentice Hall of India Pvt. Ltd, New Delhi, India. 
Jordan M. 2004 - The Encyclopedia of Fungi of Britain and Europe. Francis Lincoln Publishers Ltd., London.

Karun NC, Sridhar KR. 2014 - A preliminary study on macrofungal diversity in an arboratum and three plantations of the southwest coast of India. Current Research in Environmental \& Applied Mycology 4, 173-187.

Karun NC, Sridhar KR. 2016 - Spatial and temporal diversity of macrofungi in the Western Ghat forests of India. Applied Ecology and Environmental Research 14, 1-21.

Kidd R. 2001 - Coastal dune management, A manual of Coastal Dune Management and Rehabilitation Techniques. NSW Department of Land and Water Conservation Coastal Unit, Newcastle.

Lantieri A, Gargano ML,Venturella G. 2009 - The sabulicolous fungi from Sicily (southern Italy), additions and critical review. Mycotaxon 110, 151-154.

Maun MA 2009. The biology of coastal sand dunes. Oxford University Press, USA.

Miller OK Jr, Lodge DJ, Baroni TJ. 2000 - New and interesting ectomycorrhizal fungi from Puerto Rico, Mona, and Guana Islands. Mycologia 92, 558-570.

Mohanan C. 2011 - Macrofungi of Kerala, Handbook \# 27. Kerala Forest Research Institute, Peechi, India.

O’Hanlon R, Harrington TJ. 2011 - Diversity and distribution of mushroom-forming fungi (Agaricomycetes) in Ireland. Biology \& Environment Proceedings of the Royal Irish Academy 111B, 1-17.

Pavithra M, Sridhar KR, Greeshma AA, Karun NC. 2016 - Spatial and temporal heterogeneity of macrofungi in the protected forests of Southwestern India. Journal of Agricultural Technology 12, 105-124.

Pecoraro L, Angelini P, Arcangeli A, Bistocchi G, Gargano ML, La Rosa A, Venanzoni R. 2014 Macrofungi in Mediterranean maquis along seashore and altitudinal transects. Plant Biosystems 148, 367-376.

Pegler D. 1990 - Kingfisher Field Guide to the Mushrooms and Toadstools of Britain and Europe. Kingfisher Publications, London.

Pegler DN, Boddy L, Ing B, Kirk PM. 1993 - Fungi of Europe: Investigation, Recording and Conservation. Royal Botanic Gardens, Kew.

Phillips R. 2006 - Mushrooms. Pan Macmillan, London.

Prasannarai K, Sridhar KR. 2001 - Diversity and abundance of higher marine fungi on woody substrates along the west coast of India. Current Science 81, 304-311.

Rodrigues RS, Mascarenhas A, Jagtap GT. 2011 - An evaluation of flora from coastal sand dunes of India: Rationale for conservation and management. Ocean and Coastal Management 54, 181-188.

Sá MCA, Baseia IG, Wartchow F. 2013 - Lactifluus dunensis, a new species from Rio Grande do Norte, Brazil. Mycosphere 4, 261-265.

Seena S, Sridhar KR. 2004 - Endophytic fungal diversity of 2 sand dune wild legumes from the southwest coast of India. Canadian Journal of Microbiology 50, 1015-1021.

Sridhar KR, Bhagya B. 2007 - Coastal sand dune vegetation: a potential source of food, fodder and pharmaceuticals. Livestock Research for Rural Development 19, Article \# 84: http://www.cipav.org.co/lrrd//rrd19/6/srid19084.htm

Sridhar KR. 2009 - Bioresources of coastal sand dunes - are they neglected? In: Coastal Environments: Problems and Perspectives (ed Jayappa KS, Narayana AC), IK International Publishing House, New Delhi, 53-76.

Sulzbacher MA, Giachini AJ, Grebenc T, Silva BDB, Gurgel FE, Loiola MIB, Neves MA, Baseia IG. 2013 - A survey of an ectotrophic sand dune forest in the northeast Brazil. Mycosphere 4, 1106-1116. 
Taiga K, Tamotsu H, Seiji T, Akitomo U. 2011 - New records of two maritime Basidiomycetes, Tulstoma striatum and Geastrum quadrifidum in coastal dune of Eastern Hokkaido. Shiretoko Museum 32, 19-24.

Takehashi S, Kasuya T, Kakishima M. 2007 - Marasmiellus mesosporus, a Marasmius-blight fungus newly recorded from sand dunes of the Japanese coast. Mycoscience 48, 407-410.

Takehashi S, Kasuya T, Takehashi C, 2012 - Agaric fungi from Ishikari sand dunes in Hakkaido, Northern Japan. Wind Museum Bulletin of Ishikari Dune 2, 23-31.

Tedersoo L, Suvi T, Beaver K, Kõljalg U. 2007 - Ectomycorrhizal fungi of the Seychelles: Diversity patterns and host shifts from the native Vateriopsis seychellarum (Dipterocarpaceae) and Intsia bijuga (Caesalpiniaceae) to the introduced Eucalyptus robusta (Myrtaceae), but not Pinus caribea (Pinaceae). New Phytologist 175, 321-333.

Vila J, Àngel F, Llimona X. 1999 - Volvariella nigrovolvacea Kosina var. dunensis Vila, Àngel et Llimona var. nov. Revista Catalana de Micologia 22, 131-134.

Wolfe BE, Richard F, Cross HB, Pringle A. 2010 - Distribution and abundance of the introduced ectomycorrhizal fungus Amanita phalloides in North America. New Phytologist 185, 803816. 